\title{
Identificación, Estabilidad y Actividad Antioxidante de las Antocianinas Aisladas de la Cáscara del Fruto de Capulí (Prunus serotina spp capuli (Cav) Mc. Vaug Cav).
}

\author{
Nelson H. Hurtado y Milena Pérez \\ Universidad de Nariño, Departamento de Química, Grupo de Investigación en Productos de Importancia \\ Biológica (GIPIB), Calle 18 Carrera 50, Campus Torobajo, San Juan de Pasto - Nariño - Colombia (e-mail: \\ nhurtado@udenar.edu.co; mile5911@hotmail.com)
}

Recibido Oct. 31, 2013; Aceptado Dic. 30, 2013; Versión final recibida Feb. 27, 2014

\begin{abstract}
Resumen
A partir de la cáscara del fruto de Capulí (Prunus serotina spp capuli (Cav) Mc. Vaug Cav) se aisló mediante maceración en metanol-ácido acético y métodos cromatográficos un extracto crudo y un extracto rico en antocianinas monoméricas (ERAs). Usando espectrometría de masas y Resonancia Magnética Nuclear, se determinaron como pigmentos mayoritarios la Cianidina-3-O-(6"-O- $\alpha$-ramnopiranosil)- $\beta$ glucopiranosido y la Cianidina-3-O- $\beta$-glucopiranosido. En los extractos se evaluó el contenido de antocianinas (AT) y la capacidad antioxidante equivalente al trolox (TEAC). Para el extracto crudo se encontró menor AT y menor TEAC, resultados que están en concordancia con el mayor contenido fenólico del ERAs. La degradación de los extractos describió una cinética de primer orden, el $\mathrm{t}_{1 / 2}$ es mayor cuando los extractos se almacenan en condiciones más ácidas $(\mathrm{pH} 3)$ y en ausencia de aire y luz.
\end{abstract}

Palabras clave: antocianinas, pigmentos, estabilidad, antioxidantes, prunus serotina.

\section{Identification, Stability and Antioxidant Activity of Anthocyanins Isolated from Fruit peel of Capulí (Prunus serotina capuli spp (Cav) Mc. Vaug Cav.)}

\begin{abstract}
From the peel of the fruit Capulí (Prunus serotina capuli spp (Cav) Mc. Vaug Cav.) was isolated by maceration in methanol - acetic acid and chromatographic methods a crude extract and monomeric anthocyanin rich extract (ERAs). Using mass spectrometry and nuclear magnetic resonance, it was determined as the major pigments cyanidin-3-O-(6"-O- $\alpha-$ rhamnopyranosyl)- $\beta$-glucopyranoside and cyanidin-3-O- $\beta$-glucopyranoside. In the extracts the content of anthocyanins (TA) and the equivalent capacity to trolox (TEAC) were evaluated. For the crude extract it was found lower values of TA and TEAC, results that are consistent with the highest phenolic content of ERAs. The degradation of the extracts described a first kinetics order; the $\mathrm{t}_{1 / 2}$ is greater when the extracts are stored in more acidic conditions $(\mathrm{pH}$ 3 ) and in the absence of air and light.
\end{abstract}

Keywords: anthocyanins, pigments, stability, antioxidants, prunus serotina. 


\section{INTRODUCCIÓN}

Los colorantes naturales para alimentos son necesarios en la industria; sin embargo, colorantes naturales rojos son escasos ya que pocos extractos naturales tienen un color rojo brillante no mezclado con otros tonos (Francis, 1989). Las antocianinas han ganado gran importancia como colorantes naturales para alimentos (Giusti et al., 2003); este tipo de pigmentos de las plantas son metabolitos de tipo fenólico, pertenecen a la familia de los flavonoides y son responsables de la mayoría de colores rojos y azules de las frutas y hortalizas. Una molécula de antocianina está constituida por una aglicona o antocianidina; la cual en su estado natural esta glicosidada por uno o más azúcares. Las diferencias en el cuerpo de la aglicona se deben al número de grupos hidroxilo y al grado de metilación de estos grupos.

Por sus características estructurales es reconocido que esta clase de compuestos son potentes antioxidantes (Noda et al., 2002; Saint-Cricq et al., 1999) con reconocidos beneficios para la salud humana (Lazze et al., 2003; Morimitsu et al., 2002; Tsada et al., 1999); por lo tanto se recomienda el consumo de alimentos que los contengan. Se sabe que cuando este tipo de alimentos se procesan (ejemplo, pasteurización) siempre ocurre alguna degradación del color original; éste color perdido podría restaurarse usando como aditivo este tipo de pigmentos naturales (Francis, 1989), los que adicionalmente pueden incrementar el tiempo de vida del producto debido a sus mencionadas propiedades antioxidantes.

Los colorantes naturales tales como las antocianinas pueden impartir varios tonos rojos al color del alimento; pero hay limitaciones en su aplicación debido a su solubilidad, su dificultad para igualar el matiz deseado, incompatibilidad con la matriz del alimento y la estabilidad del color con respecto al pH, luz y oxígeno (Bridle et al., 1997; Markakis et al., 1992). Se sabe muy bien que las propiedades de las antocianinas, incluyendo la expresión del color, están altamente influenciadas por la estructura de la antocianina y por el pH (Cabrita et al., 2000). Se ha demostrado que en la región de pH entre 5-7 las antocianinas más simples son inestables y rápidamente pierden color debido a la hidratación de la posición2 del esqueleto de la antocianidina, generalmente se acepta que las antocianinas muestran su color más intenso cuando están en la forma de ion flavilio (Francis, 1989); su color rojo es el resultado neto de todas las antocianinas monoméricas, oligoméricas y poliméricas junto con sus formas copigmentadas.

En los últimos años se ha incrementado la información con respecto a las características y estabilidad de los extractos de antocianinas de diferentes frutas; por ejemplo, se ha establecido que la acilación mejora la estabilidad de las antocianinas a través de la copigmentación intramolecular (Brouillard et al., 1983). En este trabajo se presenta información con respecto al contenido fenólico, actividad antioxidante, estabilidad y caracterización estructural de las antocianinas presentes en la cáscara del fruto de Capulí. El Capulí cuyo nombre científico es Prunus serotina capuli spp (Cav) Mc. Vaug Cav pertenece a la familia de las Rosaceae; así como la ciruela, cereza, melocotón, almendro y muchas otras especies que se hallan distribuidas en diferentes regiones de América tropical. El árbol crece en forma silvestre, tiene de 5 a $15 \mathrm{~m}$ de altura y un diámetro a la altura del pecho de hasta $1.2 \mathrm{~m}$. Su fruto es redondo; con diámetro de 1 centímetro en promedio, de color verde que se torna morado al llegar a la madurez. Aunque la planta crece en varios países tropicales; ésta es relativamente abundante en Colombia y su fruto es consumido fresco o preparado en forma de jugos o mermelada casera. En la literatura científica se reportan diferentes trabajos sobre esta planta, se ha encontrado que las hojas y flores de la planta contienen compuestos fenólicos que le confieren gran poder antioxidante principalmente asociado a algunos efectos terapéuticos (tratamiento de la hipertensión) (Ibarra et al., 2009). Se ha encontrado además que las semillas del fruto son ricas en ácido oleico (Alveano et al., 2011). Asimismo, el fruto de Capulí puede usarse como un alimento funcional potencialmente útil en la prevención y tratamiento de la hipertensión (Luna et al., 2013). Son pocas las publicaciones sobre la composición del colorante extraído de la cáscara (antocianinas), Ordaz et al. (1999) ha establecido la presencia, en un extracto (acetona-agua) de la cáscara, de cianidina-3-rutinosido, cianidina-3-glucosido y un tercer pigmento posiblemente de tipo rutinósido en el fruto de Capulí de México. Luna et al. (2013) identificaron en el extracto acuoso de la cáscara cianidina-3-rutinósido. Así, el objetivo de este trabajo fue purificar, caracterizar químicamente, evaluar la capacidad antioxidante y valorar su estabilidad con respecto al $\mathrm{pH}$; en presencia y ausencia de luz y aire, de diferentes extractos del fruto de Capulí.

\section{MATERIALES Y MÉTODOS}

\section{Material vegetal}

Los frutos maduros de Capulí se recolectaron en el municipio de Pasto, Nariño, Colombia; la cosecha se realizó teniendo en cuenta las características visuales del fruto: color (cáscara $100 \%$ morada, pH 4.67), forma (globosos y lisos) y consistencia (carnosa). Un ejemplar fue codificado como 45170 en el Herbario de la Universidad de Nariño. La pulpa y la cáscara se separaron manualmente. 


\section{Aislamiento de los extractos}

Los frutos frescos y maduros se lavaron y posteriormente se aisló manualmente la cáscara de la pulpa; luego la cáscara se maceró por 8 horas en metanol-ácido acético $(19: 1, \mathrm{v} / \mathrm{v})$, se centrifugó durante 10 min a $10.000 \mathrm{rpm}$ y se filtró en un embudo Buchner, usando como medio filtrante papel con poro $11 \mu \mathrm{m}$. El solvente se removió bajo vacío a $40{ }^{\circ} \mathrm{C}$ y el residuo se liofilizó (extracto crudo, EC); este extracto se diluyó en agua $(1: 1 \mathrm{w} / \mathrm{w})$ y porciones separadas de $100 \mathrm{~g}$ se cargaron sobre una columna abierta que contenía como fase estacionaria un absorbente polimérico (Amberlita XAD-7) no iónico de dimensiones $80 \times 4 \mathrm{~cm}$ d.i. Los pigmentos absorbidos se lavaron con agua destilada y se eluyeron con $1 \mathrm{~L}$ de metanol-ácido acético (19:1 $\mathrm{v} / \mathrm{v}$ ); de acuerdo con el procedimiento descrito por Degenhardt et al (2000), el eluente se concentró al vacío a $40^{\circ} \mathrm{C}$ y la fase acuosa se liofilizó. El producto final fue un extracto enriquecido en antocianinas (ERAs).

Por último, con el fin de separar las antocianinas monoméricas y poliméricas del ERAs se utilizó el procedimiento descrito por Henke (1996). La muestra se disolvió en metanol, a continuación esta solución fue sometida a cromatografía de exclusión por tamaño utilizando una columna empacada con Sephadex LH-20 Sigma $(40 \times 3 \mathrm{~cm})$. La fracción de compuestos de bajo peso molecular (extracto rico en antocianinas monoméricas, ERAMs) se eluyó con una mezcla de metanol-agua (1:1) que contenía ácido trifluoroacético al $0.1 \%$ y la fracción polimérica fue eluída con una mezcla de acetona-agua (2:1); con ácido trifluoroacético al $0.1 \%$. La fracción monomérica se analizó por cromatografía líquida de alta eficiencia (HPLC) y una posterior purificación se llevó a cabo usando HPLC preparativa.

\section{Cuantificación de Antocianinas y Fenoles Totales}

Los fenoles totales (FT) se determinaron por el método de Folin-Ciocalteu (FC). Se midió la absorbancia a $765 \mathrm{~nm}$ y los FT se expresaron como gramos de ácido gálico por 100 gramos de cáscara (\% p/p). Para evaluar el contenido de antocianinas monoméricas se utilizó el método del pH diferencial (Giusti y Wrolstad, 2003). El contenido de pigmento fue calculado como cianidina 3-glucósido; usando un coeficiente de extinción de $26900 \mathrm{~L} / \mathrm{cm}$ x mol y un peso molecular de $449.2 \mathrm{~g} / \mathrm{mol}$. Para realizar las mediciones espectrales se utilizó un espectrofotómetro Pharo Merck.

\section{Determinación de la Capacidad Antioxidante Total}

La capacidad antioxidante total se determinó por el ensayo TEAC (Re et al., 1999). El ensayo se basa en la habilidad de los antioxidantes para capturar el mono catión radical 2,2'-azinobis-(3-etilbenzotiazolina-6-ácido sulfónico) (ABTS $\left.{ }^{\bullet}\right)$, como compuesto de referencia se usó Trolox; un análogo de la vitamina $E$ soluble en agua, con una capacidad antioxidante de $1 \mathrm{mmol}$ de Trolox/mmol de muestra (por definición).

\section{HPLC con Detector de Arreglo de Diodos}

Para el análisis de la muestra ERAMs se usó un sistema HPLC (Waters 1525) equipado con un detector de arreglo de fotodiodos (2998) y un sistema de bomba binaria (Waters 1225). La separación se realizó usando una columna X-Terra RP-18 $(100 \times 4.6 \mathrm{~mm})$ con un tamaño de partícula de $3,5 \mu \mathrm{m}$. El sistema de solventes fue acetonitrilo-ácido fórmico-agua 3:10:87, v/v/v (solvente $A$ ) y 50:10:40, v/v/v (solvente B); con una velocidad de flujo de $0.8 \mathrm{ml} / \mathrm{min}$. Para el análisis se utilizó un gradiente lineal de: 6 hasta $20 \% \mathrm{~B}$ en $0-10 \mathrm{~min}$, de 10 hasta $40 \%$ B desde $10-20 \mathrm{~min}$, de 40 hasta $50 \%$ B desde $20-30 \mathrm{~min}$, de 50 hasta $6 \%$ B de 30-35 min. El volumen de inyección fue de $20 \mu \mathrm{L}$ y la información espectral se registró sobre el rango de longitud de onda de 210 a $650 \mathrm{~nm}$. Las muestras se filtraron a través de un filtro de polipropileno $0.45 \mu \mathrm{m}$. La purificación de los pigmentos mayoritarios del ERAMs se realizó por HPLC modo preparativo utilizando el mismo equipo descrito anteriormente, para esto se usó la columna cromatográfica Geminix C-18 $(25 \times 1 \mathrm{~cm}, 5 \mu \mathrm{m})$, la mejor separación se obtuvo usando una mezcla de las fases móviles A y B en relación 70:30 a un flujo de 2 $\mathrm{mL} / \mathrm{min}$. El volumen de inyección fue de $200 \mu \mathrm{L}$ y la concentración de la muestra fue de $20 \mathrm{mg} / \mathrm{mL}$.

\section{Espectrometría de Masas-Ionización Electroespray}

El análisis de masas se realizó en un espectrómetro de masas Shimadzu QP-8000 $\alpha$; equipado con una interface electroespray. El voltaje electroespray aplicado fue de $4.5 \mathrm{kV}$, flujo del gas nebulizador de 4.5 $\mathrm{L} / \mathrm{min}$, voltaje de la sonda $4.5 \mathrm{kV}$, el voltaje del $\mathrm{CDL}$ fue de $30 \mathrm{~V}$, temperatura del $\mathrm{CDL} 230{ }^{\circ} \mathrm{C}$, voltaje del deflector $60 \mathrm{~V}$, rango $\mathrm{m} / \mathrm{z}$ de adquisición $50-800 \mathrm{u}$. La solución de las antocianinas se inyectó directamente dentro del sistema a una velocidad de flujo de $0.1 \mathrm{ml} / \mathrm{min}$.

\section{Resonancia Magnética Nuclear de las Antocianinas}

Las antocianinas aisladas se disolvieron en una mezcla de metanol-d $d_{4}-T F A-d_{1}(19: 1, v / v)$ y los espectros de RMN ${ }^{1} \mathrm{H}$ y ${ }^{13} \mathrm{C}$, COSY (correlation spectroscopy), HMBC (heteronuclear multiple bond coherence), HSQC (heteronuclear single-quantum correlation) se midieron en un equipo Bruker AMX-500. 


\section{Estudio de la Estabilidad}

Para valorar la estabilidad de este tipo de extractos se implementó la metodología publicada por Janna et al (2007). Se prepararon soluciones acuosas de cada uno de los extractos ajustando la concentración de cada una de ellas a un valor de absorbancia de $\sim 0.9$, porciones de estas soluciones se sometieron a almacenamiento a una temperatura de $18{ }^{\circ} \mathrm{C}$ en diferentes valores de $\mathrm{pH}(\mathrm{pH}=3$ y 5); ajustando el $\mathrm{pH}$ con soluciones buffer de citrato-fosfato y en presencia y ausencia de luz y aire (cada condición se evaluó por triplicado).

\section{Análisis estadístico}

El análisis estadístico se realizó por medio del software Statistica v. 6.0. En este estudio todos los análisis se evaluaron por triplicado $(\mathrm{n}=3)$ y se usó análisis de regresión lineal para obtener la velocidad de degradación $(k)$. Los valores P menores que 0.05 indican diferencias significativas entre las muestras.

\section{RESULTADOS Y DISCUSIÓN}

A partir de $1 \mathrm{Kg}$ de fruto se aisló manualmente la cáscara y se determinó que correspondía al $17.5 \%$ del fruto. Se encontró además que la fracción de cáscara soluble en metanol-ácido acético (19:1) era de 15.03 \% (extracto crudo, EC) y la fracción retenida sobre la resina XAD-7 (extracto rico en antocianinas., ERAs) fue del $6.4 \%$ de la cáscara. El análisis de los fenoles totales (FT) de los extractos fue de $1382.5 \pm 165 \mathrm{mg}$ de ácido gálico (GAE)/100 g de cáscara para el EC (242 mg GAE/100 g de fruta) y de $8099.4 \pm 81 \mathrm{mg}$ GAE/100 g de cáscara para ERAs (1417.4 mg GAE/100 g de fruta), de acuerdo con esta información es evidente la capacidad de la resina para retener los polifenoles de la cáscara. Estos resultados del contenido de fenoles son similares a los reportados por Vasco et al. (2008), quien determinó un contenido de compuestos fenólicos en la cáscara de Capulí de 1494 mg GAE/100 g de cáscara (fracción soluble en metanol al 50\%; extracto crudo).

Comparando el contenido fenólico/100 g de fruta de la fracción soluble en solvente polar (EC) con otros frutos, se considera que el fruto de Capulí puede ser una importante fuente de polifenoles, esto debido a que su contenido es mayor al publicado para extractos crudos (fracción soluble en metanol-agua) de diferentes pulpas de frutos comerciales; medido igualmente mediante el método de Folin-Ciocalteau: guayaba ( $83.0 \pm 0.1 \mathrm{mg} \mathrm{GAE} / 100 \mathrm{~g}$ de pulpa), fresa (132.1 $\pm 3.8 \mathrm{mg} \mathrm{GAE} / 100 \mathrm{~g}$ de pulpa), uva (117.1 \pm 0.6 $\mathrm{mg} \mathrm{GAE} / 100 \mathrm{~g}$ de pulpa) y azai (136.8 $\pm 0.4 \mathrm{mg} \mathrm{GAE} / 100 \mathrm{~g}$ de pulpa) (Kuskoski et al., 2005). Por otro lado, la concentración de fenoles de la cáscara del fruto de Capulí es consistente con el contenido de antocianinas totales (AT) determinado mediante el método de $\mathrm{pH}$ diferencial, se encontró que el EC contiene $2.67 \pm 0.02 \mathrm{mg}$ de Cy-3-glu/g de cáscara, mientras que el ERAs contiene $13.4 \pm 0.1 \mathrm{mg}$ de Cy-3glu/g de cáscara. Ordaz et al. (1999) obtuvieron resultados similares de contenido de antocianinas en cáscara de Capulí, los autores publican para la fracción soluble en acetona al 70\%, un valor de 2.72 mg Cy3-glu/g de cáscara.

El análisis de la capacidad antioxidante (TEAC) de los extractos (EC y ERAs) mostró que el ERAs tiene mayor capacidad para capturar el radical ABTS ( 5 veces mayor que el extracto crudo, EC) (Tabla 1); lo cual es de esperarse ya que como se mencionó anteriormente este extracto presenta el más alto contenido de antocianinas totales (AT) y fenoles totales (FT). El valor TEAC para el extracto EC del fruto de Capulí fue de $100.5 \pm 4.3 \mu \mathrm{mol}$ Trolox/g cáscara $(17.6 \mu \mathrm{mol}$ Trolox/g de fruto) siendo mayor que el reportado para otras frutas: mora $(7.10 \pm 0.22 \mu \mathrm{mol}$ Trolox/g de fruto $)$, guayaba $(8.23 \pm 0.40 \mu \mathrm{mol}$ Trolox/g de fruto) y uva $(9.21 \pm 0.20 \mu \mathrm{mol}$ Trolox/g de fruto) (Kuskoski et al., 2005); por lo que este fruto podría ser una interesante fuente de compuestos antioxidantes útiles en la industria de alimentos, farmacéutica o cosmética. La funcionalidad del método utilizado para medir la capacidad antioxidante se verificó evaluando el valor TEAC de un antioxidante conocido (ácido ascórbico); el dato experimental fue de $0.99 \pm 0,07 \mathrm{mmol}$ Trolox $/ \mathrm{mmol}$ de compuesto, que es consistente con lo publicado en la literatura: $0.99 \pm 0.10$ y $1.05 \pm 0.03 \mathrm{mmol}$ Trolox / mmol de compuesto (Re et al., 1999, Van Der Berg., 1999). Los ensayos de FT, AT y TEAC se realizaron por triplicado y se expresan como el valor \pm desviación estándar.

La purificación de los compuestos mayoritarios a partir del ERAMs se realizó mediante HPLC en modo preparativo; este proceso se simplificó en gran medida debido a la menor complejidad de este extracto aislado mediante cromatografía de exclusión por tamaño. En la figura 1 se muestra el cromatograma HPLC a $520 \mathrm{~nm}$ del ERAMs. En el cromatograma se observan dos antocianinas mayoritarias, los picos 1 y 2 (tiempos de retención de 4.7 y $5.4 \mathrm{~min}$ ) que representan $\sim 98.8 \%$ del área total. 


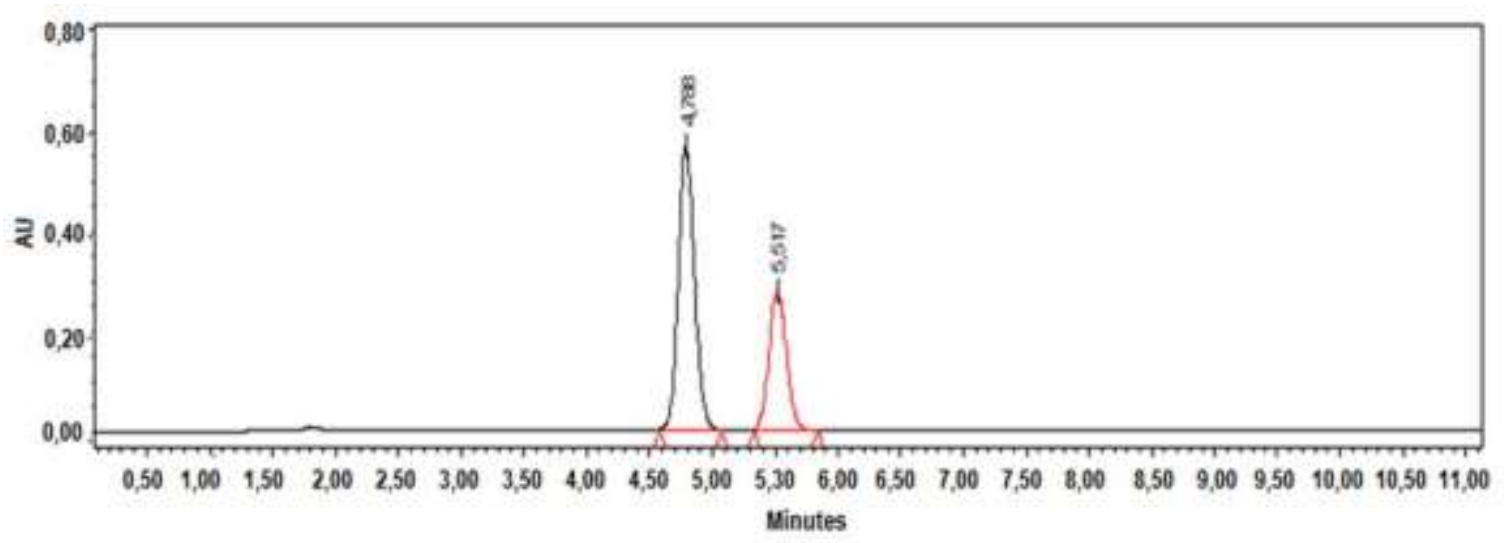

Fig. 1: Cromatograma HPLC del extracto rico en antocianinas monoméricas (ERAs). Columna X-Terra RP18, detección a 520 nm (Los números corresponden a los presentados en la tabla 1).

\section{Identificación de Antocianinas}

Después de purificar los compuestos mayoritarios se registraron los espectros UV-Vis en el rango de 260$700 \mathrm{~nm}$ y se determinaron diferentes relaciones de absorbancia (Díaz et al., 2006) que aportaron información estructural de los dos compuestos aislados. Los máximos de absorción para las antocianinas 1 y 2 fueron 515 y $517 \mathrm{~nm}$ respectivamente, la ausencia de absorción en la región 310-335 nm permite afirmar que en ninguna de las dos moléculas existe acilación; además, los dos pigmentos presentan una relación $\mathrm{A}_{440} / \mathrm{A}_{\text {vis-máx }}$ mayor de $30 \%$ que evidencia una sustitución en la posición $\mathrm{C}_{3}$ de las moléculas (Wrolstad, 2005), (tabla 1).

De acuerdo con los resultados del análisis ESI-MS la antocianina 1 tiene un peso molecular de 595u; asimismo, la presencia del ion $\mathrm{m} / \mathrm{z} 287 \mathrm{u}$ permite postular que este compuesto tiene como aglicona a la cianidina. El ion correspondiente a la aglicona se genera por la pérdida de $308 \mathrm{u}$ a partir del ion molecular; esta pérdida puede corresponder a la eliminación de dos unidades de azúcar, una de 162u (posiblemente glucosa) y otra de $146 u$ (posiblemente una ramnosa o un grupo cumaroil). En el espectro de masas se observa además un ion a m/z 449u generado por una pérdida de 146u (una ramnosa o grupo cumaroil), de acuerdo a lo discutido anteriormente se descarta la presencia del grupo acilo debido a la ausencia del máximo de absorción en el intervalo de 310-335 nm. El análisis del espectro de masas del compuesto 2 mostró un peso molecular de $449 \mathrm{u}$ y un fragmento en $\mathrm{m} / \mathrm{z} 287 \mathrm{u}$ que puede corresponder igualmente a una cianidina; este ion se genera por la pérdida de 162u (posiblemente una unidad de glucosa) (Tabla 1). De esta forma se sugiere que las dos antocianinas presentan las siguientes características estructurales: a) los pigmentos tienen como aglicona la cianidina; b) los pigmentos no tiene en su estructura grupos acilo; c) los pigmentos están sustituidos en la posición $\mathrm{C}_{3}$ de la molécula.

Tabla 1: Datos espectroscópicos, ESI-MS y TEAC de fracciones aisladas del fruto de Capulí (Prunus cerotina spp capuli (Cav) Mc. Vaug Cav)

\begin{tabular}{|c|c|c|c|c|c|c|}
\hline \multirow[t]{2}{*}{ Muestra } & \multirow{2}{*}{$\begin{array}{c}\text { Antocianinas } \\
\text { (mg C3G/g de } \\
\text { cáscara) }\end{array}$} & \multirow[t]{2}{*}{$\begin{array}{l}\lambda \max \\
(\mathrm{nm})\end{array}$} & \multicolumn{2}{|c|}{$\begin{array}{l}\text { Relación de } \\
\text { absorbancias }\end{array}$} & \multirow{2}{*}{$\begin{array}{c}\text { TEAC } \\
\mu \text { mol Trolox / g } \\
\text { cáscara }\end{array}$} & \multirow[t]{2}{*}{$\begin{array}{l}\text { Espectrometría de masas } \\
\qquad \text { ESI-MS }(\mathrm{m} / \mathrm{z})\end{array}$} \\
\hline & & & $\lambda_{440 / 520}$ & $\lambda_{320 / 520}$ & & \\
\hline $\mathrm{EC}$ & $2.67 \pm 0.02$ & - & - & - & $100,5 \pm 4.3$ & - \\
\hline ERAs & $13.4 \pm 0.1$ & - & - & - & $499.3 \pm 24.6$ & - \\
\hline $\begin{array}{c}\text { Pigmento } \\
1\end{array}$ & - & 515 & 0.319 & 0.091 & - & $\begin{array}{l}595[\mathrm{M}]+, \quad 449[\mathrm{M}-162]+287 \\
{[\mathrm{M}-162-146]+}\end{array}$ \\
\hline $\begin{array}{l}\text { Pigmento } \\
2\end{array}$ & - & 517 & 0.310 & 0.092 & - & $449[\mathrm{M}]+, 287[\mathrm{M}-162]+$ \\
\hline
\end{tabular}

Para confirmar estas características estructurales y elucidar completamente las moléculas, los dos compuestos se analizaron por espectroscopia de Resonancia Magnética Nuclear (RMN). En la parte del campo bajo del espectro de $\mathrm{RMN}{ }^{1} \mathrm{H}$ del compuesto 1 se observan seis resonancias $(8.04,7.03,8.30,6.89$, $6.68,8.94 \mathrm{ppm}$ ) (Figura 2). 


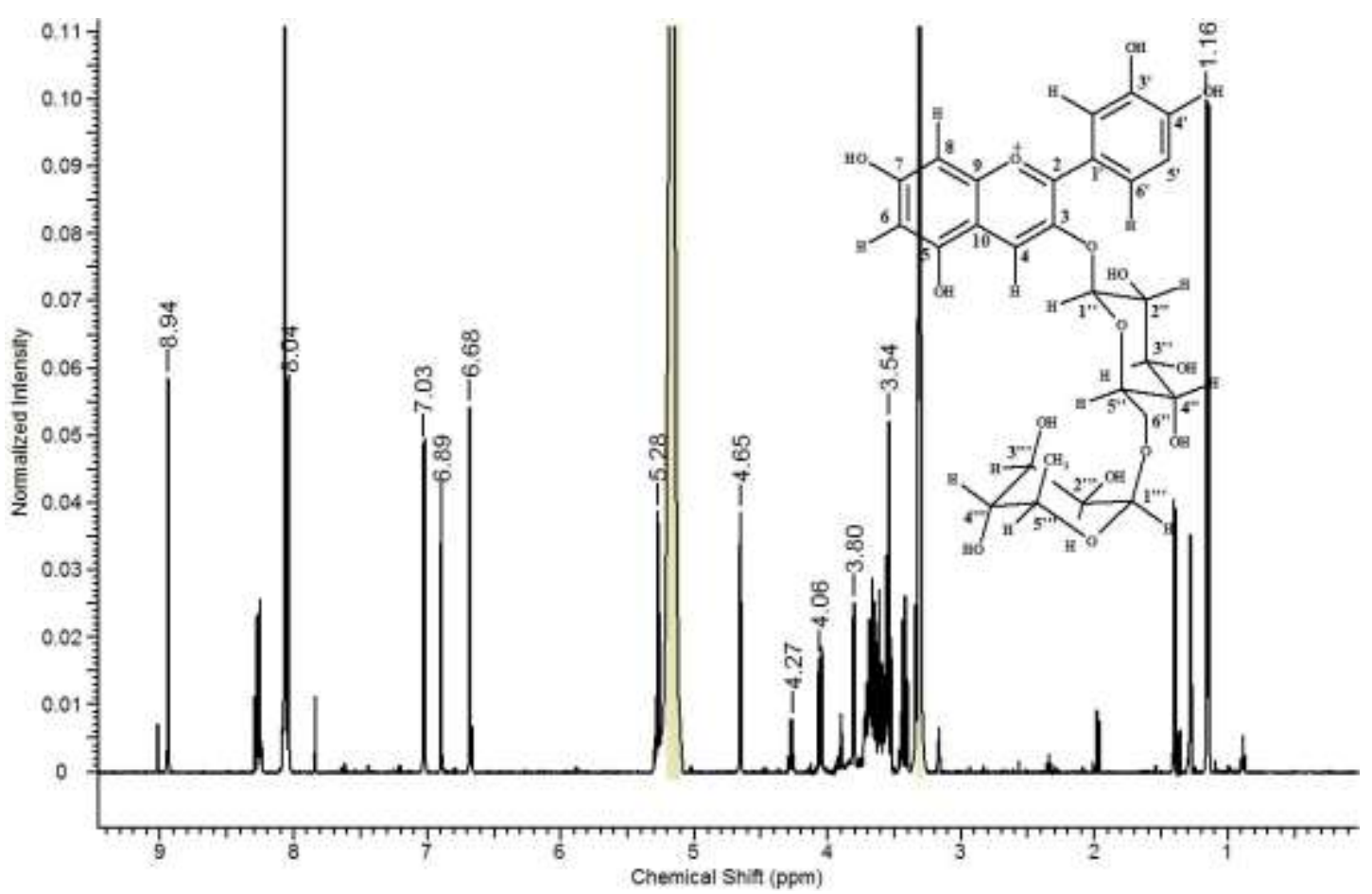

Fig. 2: Espectro RMN-1 ${ }^{-1}$ de la antocianina 1 aislada del fruto de Capulí. Bruker AMX-500, metanol-d4-TFA$d_{1}(19: 1, v / v)$.

Con base en el desplazamiento químico y patrones de acoplamiento, la señal a $8.04,7.03$ y $8.30 \mathrm{ppm}$ se asignó a H-2', H-5' y H-6' respectivamente (Figura 2). Se asignaron los $2 \mathrm{H}$ a 6.89 y 6.68 como H-8 y H-6, y el singlete a $8.94 \mathrm{ppm}$ como $\mathrm{H}-4$. Los desplazamientos químicos del ${ }^{13} \mathrm{C}$ y el experimento HSQC confirmó la identidad de la aglicona como cianidina. Mediante el análisis de los espectros COSY, HSQC y HMBC fue posible asignar las resonancias ${ }^{1} \mathrm{H} \mathrm{y}{ }^{13} \mathrm{C}$ de los azúcares, los cuales se identificaron como unidades glucopiranosil y ramnopiranosil; además, se estableció por HMBC el sitio de enlace de los azúcares a la aglicona, correlaciones en el espectro HMBC de esta molécula a 5.28/144.2 ppm (H1"/C3) y 4.65/68.4 (H1'"/C6") muestran que una unidad de glucosa está unida a la aglicona en la posición 3 y la unidad de ramnosa está unida a la glucosa en la posición 6" (Figura 2). La configuración $\beta$ de la glucosa se confirmó por la magnitud de la constante de acoplamiento $J_{1}{ }^{\prime \prime} 2^{\prime \prime}(J=7.8 \mathrm{~Hz})$ en el espectro RMN ${ }^{1} \mathrm{H}$. Experimentos HSQC y las constantes de acoplamiento están de acuerdo con una unión $\alpha$-ramnopiranosil. Los datos UVVis, HPLC-MS y RMN del pigmento 1 están en concordancia con la Cianidina-3-O-(6"-O- $\alpha$-ramnopiranosil)$\beta$-glucopiranósido (Figura 2). De la misma forma se encontró que la identidad del compuesto 2 corresponde a la molécula Cianidina-3-O- $\beta$-glucopiranósido.

Diferentes autores han investigado las propiedades bioactivas de estos dos compuestos mayoritarios identificados en la cáscara del fruto de Capulí. En ensayos in vivo se ha encontrado que la Cianidina-3-O- $\beta$ glucopiranósido actúa como agente cardioprotector (Ziberna et al., 2012), antiinflamatorio (Wang et al., 2008), anti-obesidad (Kaume et al., 2012), protector de lesiones gástricas (Li et al., 2008) y como protector contra el estrés oxidativo (Song et al., 2013). Adicionalmente, Chen et al. (2006) establecieron que la Cianidina-3-O-(6"-O- $\alpha$-ramnopiranosil)- $\beta$-glucopiranósido y la Cianidina-3-O- $\beta$-glucopiranósido inhiben el cáncer de pulmón. Estos estudios muestran que la cáscara de la fruta en estudio puede usarse como un alimento funcional, que puede ser potencialmente útil en la prevención y tratamiento de diversas enfermedades.

\section{Estudio de la estabilidad}

Para estudiar la cinética de degradación de extractos acuosos de las antocianinas (EC y ERAs) del fruto de Capulí, durante un periodo de almacenamiento de 90 días, en diferentes condiciones de almacenamiento; pH 3 y 5 y en presencia y ausencia de aire (PL; con luz, AL; sin luz, PA; con aire, AA; sin aire), se graficó el logaritmo de la relación de absorbancias $A_{t} / A_{\circ}$ en función del tiempo. Cada tratamiento se realizó por triplicado y se usó análisis de regresión lineal para obtener la velocidad de degradación $(k)$, los datos se expresan como el valor \pm desviación estándar (Figura 3). La relación lineal indica que la degradación de las antocianinas en las condiciones de almacenamiento sigue una cinética de orden uno. Estos resultados están de acuerdo con investigaciones previas en las que se demuestra que la degradación de las antocianinas de diversas matrices describen una cinética de reacción de primer orden (Sagdic et al., 2013; Jiménez et al., 2012). 


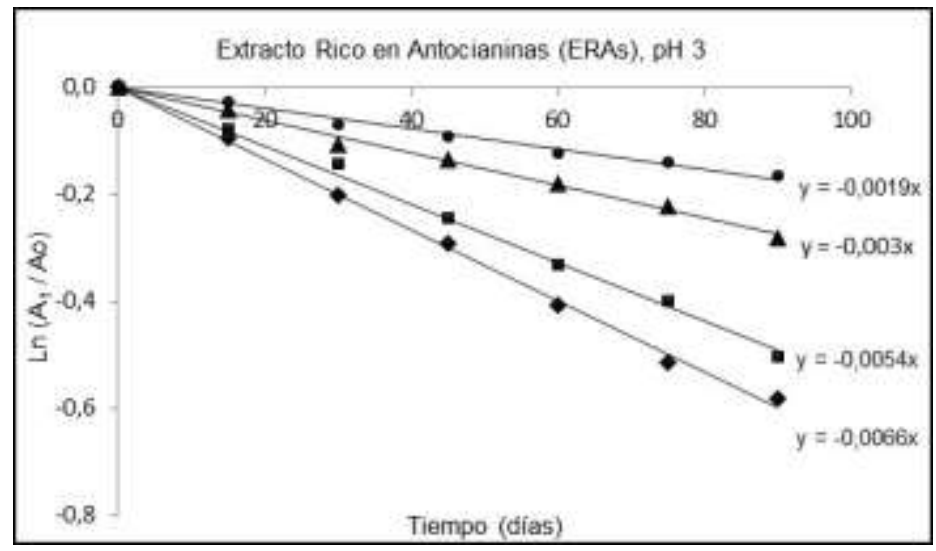

\begin{tabular}{|c|c|c|}
\hline Muestra & $\mathrm{t}_{1 / 2}$ (días) & $k\left(10^{-3}\right.$ días $\left.^{-1}\right)$ \\
\hline $\mathrm{PA}, \mathrm{PL}$ & $105.0 \pm 0.40$ & $6.6 \pm 0.38$ \\
\hline - $\mathrm{PA}, \mathrm{AL}$ & $128.4 \pm 0.58$ & $5.4 \pm 0.45$ \\
\hline $\boldsymbol{A} \mathrm{AA}, \mathrm{PL}$ & $231.0 \pm 0.45$ & $3.0 \pm 0.98$ \\
\hline - $\mathrm{AA}, \mathrm{AL}$ & $364.8 \pm 0.98$ & $1.9 \pm 0.87$ \\
\hline
\end{tabular}

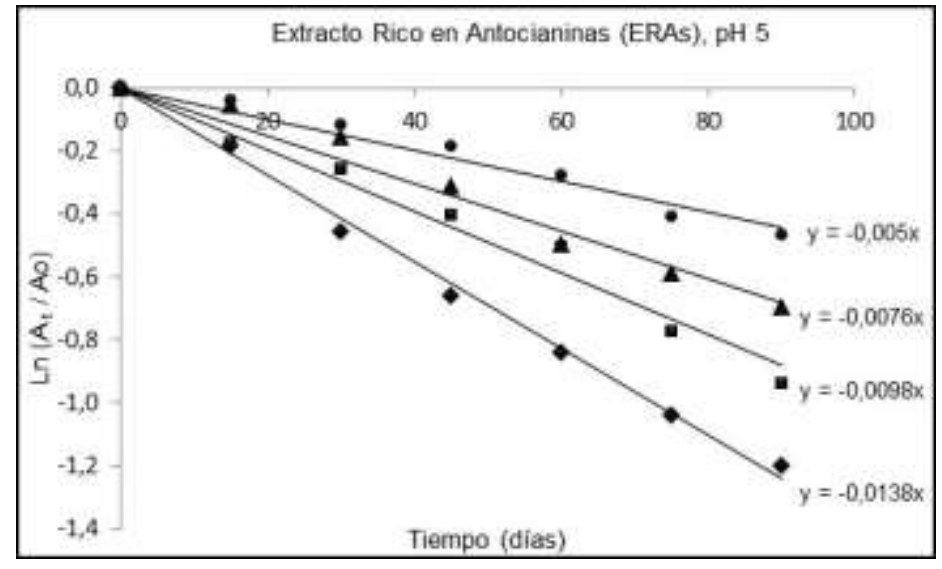

\begin{tabular}{|c|c|c|}
\hline Muestra & $t_{1 / 2}$ (días) & $k\left(10^{-3}\right.$ días $\left.^{-1}\right)$ \\
\hline - PA, PL & $50.2 \pm 0.82$ & $13.8 \pm 0.48$ \\
\hline - $\mathrm{PA}, \mathrm{AL}$ & $70,7 \pm 0.74$ & $9.8 \pm 0.45$ \\
\hline$\Delta \mathrm{AA}, \mathrm{PL}$ & $91.2 \pm 0.32$ & $7.6 \pm 0.89$ \\
\hline - $\mathrm{AA}, \mathrm{AL}$ & $138.6 \pm 0.48$ & $5.0 \pm 0.57$ \\
\hline
\end{tabular}

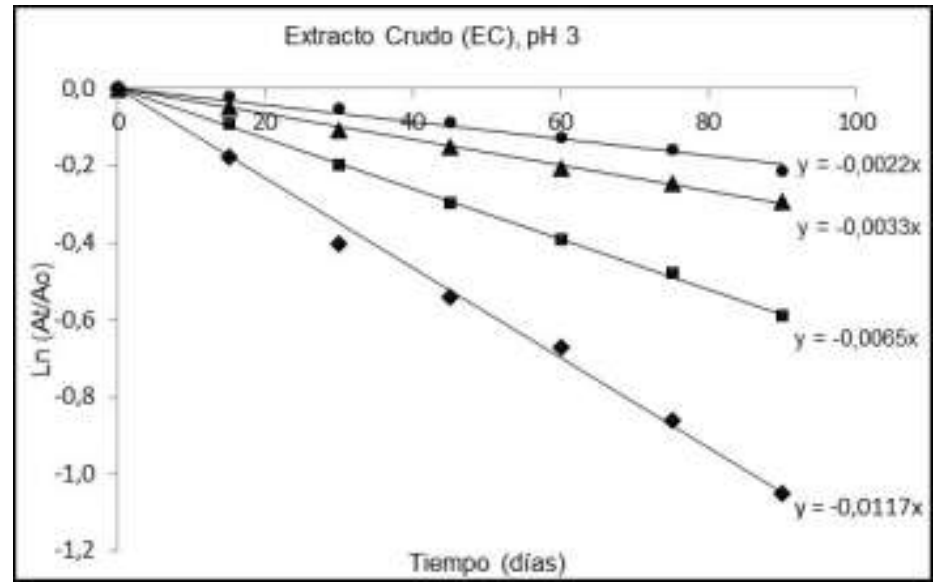

\begin{tabular}{|c|c|c|}
\hline Muestra & $\mathrm{t} 1 / 2$ (días) & $k\left(10^{-3}\right.$ días $\left.^{-1}\right)$ \\
\hline PA, PL & $59.2 \pm 0.90$ & $11.7 \pm 0.06$ \\
\hline - $\mathrm{PA}, \mathrm{AL}$ & $106.6 \pm 1.35$ & $6.5 \pm 0.05$ \\
\hline $\mathbf{A} \mathrm{AA}, \mathrm{PL}$ & $209.8 \pm 3.95$ & $3.3 \pm 0.12$ \\
\hline - $\mathrm{AA}, \mathrm{AL}$ & $315.1 \pm 10.5$ & $2.2 \pm 0.06$ \\
\hline
\end{tabular}

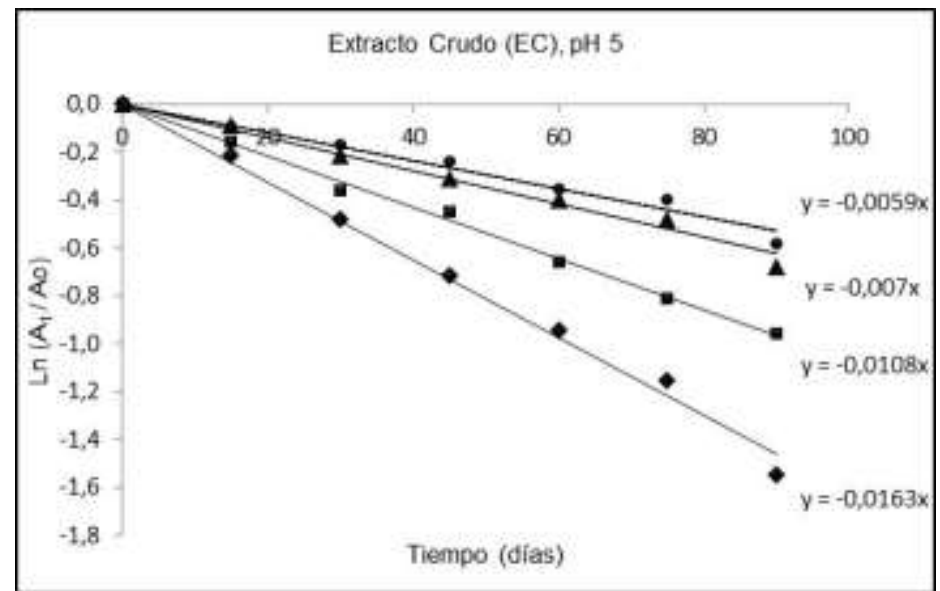

\begin{tabular}{|c|c|c|}
\hline Muestra & $\mathrm{t}_{1 / 2}$ (días) & $k\left(10^{-3}\right.$ días $\left.^{-1}\right)$ \\
\hline PA, $\mathrm{PL}$ & $42.5 \pm 0.21$ & $16.3 \pm 0.34$ \\
\hline - $\mathrm{PA}, \mathrm{AL}$ & $64.2 \pm 0.54$ & $10.8 \pm 0.49$ \\
\hline A $\mathrm{AA}, \mathrm{PL}$ & $99.0 \pm 0.06$ & $7.0 \pm 0.87$ \\
\hline - $\mathrm{AA}, \mathrm{AL}$ & $117.5 \pm 0.27$ & $5.9 \pm 0.12$ \\
\hline
\end{tabular}

Fig. 3: Comportamiento cinético del proceso de degradación del EC y ERAs en diferentes condiciones de almacenamiento (PA; presencia de aire, PL; presencia de luz, AL; ausencia de luz, AA; ausencia de aire). 
La cinética para este tipo de reacción se expresó mediante las siguientes ecuaciones:

$\ln \left(\frac{A_{t}}{A_{0}}\right)=-k^{*} t$

$\mathrm{t}_{1 / 2}=-\ln 0.5 / \mathrm{k}$

Dónde: $A_{t} / A_{o}=$ relación de absorbancias medidas a $\lambda_{\max } ; A_{o}=$ absorbancia inicial de la solución; $A_{t}=$ absorbancia medida después de un tiempo de reacción; $\mathrm{t}=$ tiempo de reacción (días); $k=$ constante de velocidad de reacción; $t_{1 / 2}=$ tiempo de vida media (días).

El tiempo de vida media de los extractos y la velocidad de degradación de las soluciones acuosas del EC y ERAs bajo diferentes tratamientos (PA, PL; PA, AL; AA, PL; AA, AL) muestra que para cada condición este parámetro es significativamente independiente al nivel de $P<0.05$. En la figura 3 se muestran los parámetros cinéticos de la degradación de las antocianinas en las diferentes condiciones de almacenamiento. En ambos extractos (EC y ERAs) al disminuir el $\mathrm{pH}(\mathrm{pH} 3)$ se disminuye la velocidad de degradación de las antocianinas como se demuestra por las constantes de velocidad $(k)$ y los tiempos de vida media $\left(\mathrm{t}_{1 / 2}\right)$. Se puede apreciar además que la luz y el aire tienen un claro efecto en la degradación de las antocianinas; tal como puede observarse por las constantes $k$ obtenidas. El almacenamiento en presencia de aire y luz (PA, PL) resultó en una mayor degradación de las antocianinas respecto a las soluciones que se almacenaron en ausencia de aire y luz ( $A A, A L)$; así, el tiempo de vida media $t_{1 / 2}$ para las soluciones del ERAs almacenadas a pH 3 en AA y AL fue de $364.8 \pm 0.98$ días y solo de $105.0 \pm 0.40$ días en presencia de aire y luz, este comportamiento se observó en todas las soluciones almacenadas en los dos valores de $\mathrm{pH}$. La mayor degradación del extracto crudo, durante el periodo de almacenamiento en presencia de aire y luz en ambos valores de pH (Figura 3), puede estar relacionada con la presencia de azúcares y proteínas que participan en reacciones de Maillard (pardeamiento enzimático) que pueden acelerar la degradación de las antocianinas (Von Elbe et al., 1996). De otro lado, entre el aire y la luz, el factor que más influye en la degradación de este tipo de extractos es el aire.

De manera general se puede advertir que de acuerdo a los valores de la constante de velocidad de reacción y el tiempo de vida media de los extractos almacenados; existe una gran influencia del $\mathrm{pH}$, la luz, el aire sobre la estabilidad de las soluciones acuosas de las antocianinas del fruto de Capulí. Está bien documentado que el pH tiene una fuerte influencia en la estabilidad de las antocianinas (Eiro y Heinonen, 2002); este tipo de compuestos cuando están en solución pueden presentar cambios de coloración, dependiendo de su estructura y valor de $\mathrm{pH}$. El incremento de $\mathrm{pH}$ hace que el catión flavilio (forma más estable) se vea expuesto a un ataque nucleofílico del agua produciendo la pseudobase carbinol, en la que se observa la pérdida de conjugación molecular. Estos resultados indican también que las matrices de antocianinas tienen un notable efecto sobre la estabilidad y que a la vez el pH puede favorecer o retrasar dicho fenómeno. Se sabe además que el aire como agente oxidante junto con la luz entre otros, pueden hacer que las antocianinas se degraden, la luz es capaz de acelerar algunos cambios fotoquímicos, ocasionando eventualmente la degradación de la antocianina hasta la forma chalcona, limitando su aplicación como aditivo alimenticio (Eiro y Heinonen, 2002).

\section{CONCLUSIONES}

De los resultados mostrados, de su análisis y de su discusión, se pueden obtener las siguientes conclusiones, sobre el extracto de polifenoles (antocianinas) aislados de la cáscara del fruto de Capulí (Prunus serotina spp capuli (Cav) Mc. Vaug Cav): 1) el análisis de los fenoles totales (FT) y la capacidad antioxidante (TEAC) de la fracción soluble en metanol-ácido acético (19:1) muestra que el fruto en estudio puede ser una potencial fuente de compuestos antioxidantes para su uso en la industria de alimentos, farmacéutica o cosmética; 2) el extracto purificado (ERAs) mostró mayor capacidad para capturar el radical ABTS que el EC; esto se debe al mayor contenido de polifenoles en este extracto producto de su enriquecimiento mediante cromatografía en columna (Amberlita XAD-7); 3) se identificaron inequívocamente la cianidina-3-O-(6"-O- $\alpha$-ramnopiranosil)- $\beta$-glucopiranósido y la cianidina-3-O- $\beta$-glucopiranósido como las antocianinas mayoritarias presentes en la cáscara del fruto de Capulí; 4) se describió una cinética de degradación de primer orden para soluciones acuosas de antocianinas del fruto de Capulí. Se dispone así por primera vez de un estudio cinético de antocianinas en extractos de Capulí; 5) el pH tiene un efecto importante en la degradación de las antocianinas y dicha degradación se hace menor a valores de pH bajo, donde se ha demostrado que las antocianinas son más estables. Además se puede concluir que en ambos valores de $\mathrm{pH}$ las muestras son más estables cuando se almacenan en ausencia de aire y luz. Entre el aire y la luz, el factor que más influye en la degradación de los extractos de Capulí es el aire; 6) La matriz donde se encuentran las antocianinas tiene un gran efecto sobre la estabilidad, la mayor degradación del extracto crudo (EC), en presencia de aire y luz y en ambos valores de $\mathrm{pH}$, puede deberse no solo con la oxidación y 
los cambios fotoquímicos relacionados con la luz, sino también con la existencia en esta fracción de azúcares y proteínas; que participan en reacciones de Maillard que aceleran la degradación de las antocianinas.

\section{AGRADECIMIENTOS}

Los autores agradecen el apoyo económico brindado por la Vicerrectoría de Investigaciones Postgrados y Relaciones Internacionales- VIPRI - UDENAR.

\section{REFERENCIAS}

Alveano, I., A. Rojas, D. Oomahb y J.C.G. Drover, Characteristics of Prunus serotina seed oil, Food Chemistry: 124(3), 983-990 (2011).

Bridle, P y C.F. Timberlake, Anthocyanins as natural food colours- selected aspects, Food Chemistry: 58(1), 103-109 (1997).

Brouillard, R, The in vivo expression of anthocyanins color in plant, Phytochemistry: 22(6), 1311-1323 (1983).

Chen, P.N., S.C. Chu, H.L. Chiou, W.H. Kuo, C.L. Chiang y Y.S. Hsieh, Mulberry anthocyanins, cyanidin 3rutinoside and cyanidin 3-glucoside, exhibited an inhibitory effect on the migration and Invasion of a human lung cancer cell line, Cancer Letters: 235(2), 248-259 (2006).

Dehenhardt, A., H. Knapp y P. Winterhalter, Separation and purification of anthocyanins by high-speed countercurrent chromatography and screening for antioxidant activity, Journal Agriculture and Food Chemistry: 48 (2), 338-343 (2000).

Díaz, L.S., C. Padilla y C. Sepúlveda, Identificación del principal pigmento presente en la cáscara del maracuyá púrpura (Passiflora edulis), Información Tecnológica: 17 (6), 75-84 (2006).

Eiro, M., M y Heinonen, M, Anthocyanin color behavior and stability during storage: Effect of intermolecular copigmentation, Journal of Agricultural and Food Chemistry: 50 (25), 7461-7466 (2002).

Francis, F.J, Food colorants: anthocyanins, Critical Reviews Food Science and Nutrition: 28(4), 273-314 (1989).

Giusti, M.M y R.E. Wrolstad, Acylated anthocyanins from edible sources and their applications in food systems, Biochemical Engineering Journal: 14(3), 217-225 (2003).

Henke, H, Preparative gel chromatography on sephadex LH-20, Journal Fur Praktische Chemie ChemikerZeitung: 338 (1), 291-295 (1996).

Ibarra, C., A. Rojas, F. Luna, I. Rojas, B. Rivero y J. Rivero, Vasorelaxant constituents of the leaves of Prunus Serotina “Capulin”, Revista Latinoamericana de Química: 37(2), 164-173 (2009).

Jiménez, N., P. Bohuon, M. Dornier, C. Bonazzina, M. Pérez y F. Vaillan, Effect of water activity on anthocyanin degradation and browning kinetics at high temperatures $\left(100-140{ }^{\circ} \mathrm{C}\right)$, Food Research International: 47 (1), 106-115 (2012).

Kaume, L., W.C. Gilbert, C. Brownmiller, L.R. Howard y L. Devareddy, Cyanidin 3-O- $\beta$-D-glucoside-rich blackberries modulate hepatic gene expression and anti-obesity effects inovariectomized rats, Journal of Functional Foods: 4(2), 480-488 (2012).

Kuskoski, M., A. Asuero, A.M. Troncoso, J. Manani-Filho y R. Fett, Aplicación de diversos métodos químicos para determinar actividad antioxidante en pulpa de frutos, Food Science and Technology: 25 (4), 726-732 (2005).

Lazze, M.C., R. Pizzala, M. Savio, L.A. Stivala, E. Prosperi y L. Bianchi, Anthocyanins protec against DNA damage induced by ter-butyl-hydroperoxide in rat smooth muscle and hepatoma cells, Mutation Research: 535(1), 103-115 (2003). 
Li, C.Y., H.D. Xu, B.T. Zhao, H. I. Chang y H.I. Rhee, Gastroprotective effect of cyanidin 3-glucoside on ethanol-induced gastric lesions in rats, Alcohol: 42(8), 683-687 (2008).

Luna, F.J., C. Ibarra, A. Rojas, J. Rojas, E. Yahia, D.M. Rivera, A. Rojas y M.A. Zavala, Nutraceutical value of black cherry Prunus serotina Ehrh. fruits: antioxidant and antihypertensive properties. Molecules: 18 (11), 14597-14612 (2013).

Markakis, P. Stability of anthocyanins in food. In Anthocyanins as food colors by P. Markakis, pp163-180 Academic Press., New York, USA (1992).

Morimitsu, Y., K. Kubota, T. Tashiro, E. Hashizume, T. Kamiyo y T. Osawa, Inhibitory effect of anthocyanins and colored rice on diabetic cataract formation in the rat lenses. International Congress Series: 1245(6), 503508 (2002).

Noda, Y., T. Kaneyuki, A. Mori y L. Packer, Antioxidant activities of pomegranate fruit extract and its anthocyanidins: delphinidin, cyaniding, and pelargonidin, Journal Agriculture and Food Chemistry: 50(1), 166-171 (2002).

Ordaz, A., P. Wesche-Ebeling, L. Wrolstad, L. Rodriquez y A. Argaiz, Purification and identification of Capulin (Prunus serotina Ehrh.) anthocyanins, Food Chemistry: 65(2), 201-206 (1999).

Re, R., N. Pellegrini, A. Proteggente, M. Pannala, C. Yang y C. Rice-Evans, Antioxidant activity applying an improved ABTS radical cation decolorization assay, Free Radical Biology and Medicine: 26 (9), 1231-1237 (1999).

Sagdic, O. y otros siete autores, Cytotoxic and bioactive properties of different color tulip flowers and degradation kinetic of tulip flower anthocyanins, Food and Chemical Toxicology: 58 (5), 432-439 (2013).

Saint-Cricq, N., Y. Glories y N. Vivas, Free radical scavenging effect of anthocyanins in red wines, Food Research International: 32(5), 327-333 (1999).

Song, J., M. Zhao, X. Liu,Y. Zhu, X. Hu, F. Chen, Protection of cyanidin-3-glucoside against oxidative stress induced by acrylamide in human MDA-MB-231cells, Food and Chemical Toxicology 58(8), 306-310 (2013).

Tsada, T., F. Hono, J. Kitoh y T. Osawa, Protective effects of dietary cyanidin 3-O- $\beta$-D-glucoside on liver ischemia-reperfusion injury in rats, Archives of Biochemistry and Biophysics: 368(2), 361-366 (1999).

Van Den Berg, R., G.R. Haenen y H. Van Den Berg, Applicability of an improved trolox equivalent antioxidant capacity (TEAC) assay for evaluation of antioxidant capacity measurements of mixtures, Food Chemistry: 66 (4), 511-517 (1999).

Vasco, C., J. Ruales y A. Kamal, Total phenolic compounds and antioxidant capacities of major fruits from Ecuador, Food Chemistry: 111(4), 816-823 (2008).

Von Elbe, J.H y S.J. Schwartz. Colorants. In Food chemistry by O. R. Fennema, pp 651-722, Marcel Dekker., New York, USA (1996).

Wang, Q., M. Xia, C. Liu, H. Guo, Q. Ye,Y. Hu,Y. Zhang, M. Hou, H. Zhu, J. Ma, W. Ling, Cyanidin-3-O- $\beta-$ glucoside inhibits iNOS and COX-2 expression by inducing liver $X$ receptor alpha activation in THP-1 macrophages, Life Sciences: 83(5-6), 176-184 (2008).

Wrolstad, R., R. Durst y J. Lee, Tracking color and pigment changes in anthocyanins products, Trends in Food Science and Technology: 16 (9), 423-428 (2005).

Ziberna, L., F. Tramera, S. Mozec, U. Vrhovsekd, F. Mattivid y S. Passamontia, Transport and bioactivity of cyanidin 3-glucoside in to the vascular endothelium, Free Radical Biology and Medicine: 52(9),1750-1759 (2012). 